\title{
Experimental determination of the Stokes parameters using a dual photoelastic modulator system
}

\author{
Wei Guan, ${ }^{1,2}$ Paul J. Cook, ${ }^{1}$ Grenville A. Jones, ${ }^{1}$ and Tiehan H. Shen ${ }^{1, *}$ \\ 'Joule Physics Laboratory, School of Computing, Science and Engineering, \\ University of Salford, Salford M5 4WT, UK \\ ${ }^{2}$ Current address: NanoLAB, Department of Engineering Materials, \\ University of Sheffield, Sheffield S1 3JD, UK \\ ${ }^{*}$ Corresponding author: t.shen@salford.ac.uk
}

Received 22 December 2009; revised 8 April 2010; accepted 15 April 2010; posted 15 April 2010 (Doc. ID 121811); published 4 May 2010

\begin{abstract}
We develop a more general methodology for a dual photoelastic modulator (PEM) system that is used for the determination of the Stokes parameters of an arbitrary light beam. This allows for a degree of arbitrariness in the setting of the retardation amplitudes of both PEMs, thus permitting a robust and effective optimization of the detection system. Various experimental issues are considered and a calibration procedure is described that ensures accurate measurement of the absolute values of the Stokes parameters. Measurements of the Faraday rotation of a CoPt multilayer film are provided as a demonstration of the sensitivity of the dual-PEM system. (C) 2010 Optical Society of America

OCIS codes: $120.2130,230.3810$.
\end{abstract}

\section{Introduction}

The introduction of the photoelastic modulator (PEM) some 40 years ago by Billardon and Badoz [1] has transformed the experimental measurement of the Stokes parameters [2,3]. The subject has continued to evolve insofar as modern practice tends to favor a dual-PEM setup [4-9] rather than the use of a single modulator [10-22]. The main advantage to be gained is that signals relevant to the four Stokes parameters may be collected simultaneously, thus allowing the measurement of rapidly time-varying polarization states [5,23].

Many key aspects of PEM-based polarimetry (both practical and experimental) were established by the pioneering work of Kemp [24]. Further important contributions were made by Kuldepp et al.[8] and Boyer et al. [9]. In a previous paper [23], we presented a generalized methodology for the use of a dual-PEM system, but subject to the condition that the retardation amplitudes of the modulators were set at a

0003-6935/10/142644-09\$15.00/0

(C) 2010 Optical Society of America specified fixed value in order to make the zero-order Bessel functions vanish. The adoption of this constraint allows for a comparatively easy analysis of the data. It also leads to a calibration procedure that gives a determination of the coefficients that relate measured signals directly to the values of the Stokes parameters. Using a detailed error analysis, it was also shown that the number of instrumental variables assigned fixed (as distinct from arbitrary) values should be kept to a minimum in order to reduce systematic error. Henceforth, we shall refer to this experimental setup as the "diagonalized case," for reasons that will emerge later.

In this paper, based on the theory previously described [23], we investigate the case in which no constraint is laid upon the retardation amplitudes of the two modulators. The retention of two additional degrees of freedom enhances the flexibility of the setup and offers several advantages compared with the diagonalized case, e.g., allowing the optimization of a particular signal recovery, if required, and stabilization with respect to changes in ambient temperature. These advantages will be discussed in this paper. A detailed description of experimental 
methods is presented, together with a more general calibration procedure. Results of a comparison between the diagonalized and the present (nondiagonalized) cases are also presented. Finally, as an illustration of its efficacy, the technique is applied to the observation of the Faraday effect of a CoPt multilayer film.

\section{Background Theory}

The disposition of relevant optical components is shown in Fig. 1. The dual-PEM detection system consists of two PĒMs, an analyzer, and the photodetector. The modulation axis of PEM2 defines the $x$ axis of the laboratory coordinate system. The angles between the $x$ axis and the modulation axis of PEM1, and between the $x$ axis and the passing axis of the analyzer, are denoted as $\alpha$ and $\beta$, respectively. The laser, polarizer, and compensator are used only to calibrate the dual-PEM system, a procedure discussed in more detail in Section 4 . The angle between the polarizer and the $x$ axis is denoted $\theta$.

For a monochromatic light beam of arbitrary polarization state passing through the two PEMs and an analyzer, the total theoretical intensity $I^{\prime}$ received at the photodetector can be written [23] as
Here, $I, Q, U$, and $V$ are the Stokes parameters of the incident light beam whose polarization state is sought. $\Omega_{1}$ and $\Omega_{2}$ are the fundamental modulation frequencies of the two PEMs. $J_{m}\left(\delta_{10}\right)$ and $J_{n}\left(\delta_{10}\right)$ represent standard Bessel functions of the first kind, with $m$ an odd integer and $n$ an even integer (excluding zero). $\delta_{10}$ is the retardation amplitude (in radians) of the first modulator and may be written as $(2 \pi / \lambda) \Delta_{10}$, where $\Delta_{10}$ is the maximum path difference (usually expressed in units of wavelength $\lambda$ ) introduced by the modulator. A similar notation pertains to the second modulator.

Four distinct signals (one dc and three ac) must be collected to ensure a complete determination of the Stokes parameters. The terms in the first four lines of Eq. (1) comprise the dc signal; this is needed to obtain $I$. Any of the terms in the ninth to twelfth lines in Eq. (1) may be used to derive $V$. To find $U$ and $Q$, it is essential to monitor a term in the fifth line associated with $\cos \left(n \Omega_{2} t\right)$, together with any one of the terms in the sixth to eighth lines. (The latter all contain the common factor $[Q \sin (2 \alpha)-U \cos (2 \alpha)]$, which makes them insufficient to determine both $U$ and $Q$.) We denote any one of the terms in the sixth to eighth lines as $I_{Q U 1}$ and in the fifth line as $I_{Q U 2}$. Taking

$$
\begin{aligned}
I^{\prime}= & \frac{1}{2} I+\frac{1}{2} Q \cos ^{2}(2 \alpha) \cos (2 \beta)+\frac{1}{4} U \sin (4 \alpha) \cos (2 \beta) \\
& +\frac{1}{2} \sin (2 \alpha) \cos (2 \beta)[Q \sin (2 \alpha)-U \cos (2 \alpha)] J_{0}\left(\delta_{10}\right) \\
& +\frac{1}{2} \sin (2 \alpha) \sin (2 \beta)[Q \cos (2 \alpha)+U \sin (2 \alpha)] J_{0}\left(\delta_{20}\right) \\
& -\frac{1}{2} \cos (2 \alpha) \sin (2 \beta)[Q \sin (2 \alpha)-U \cos (2 \alpha)] J_{0}\left(\delta_{10}\right) J_{0}\left(\delta_{20}\right) \\
& +\left\{\sin (2 \alpha) \sin (2 \beta)\left[1-J_{0}\left(\delta_{10}\right)\right][Q \cos (2 \alpha)+U \sin (2 \alpha)]+U \sin (2 \beta) J_{0}\left(\delta_{10}\right)\right\} \sum_{n}^{\infty} J_{n}\left(\delta_{20}\right) \cos \left(n \Omega_{2} t\right) \\
& +\left[\sin (2 \alpha) \cos (2 \beta)-\cos (2 \alpha) \sin (2 \beta) J_{0}\left(\delta_{20}\right)\right][Q \sin (2 \alpha)-U \cos (2 \alpha)] \sum_{n}^{\infty} J_{n}\left(\delta_{10}\right) \cos \left(n \Omega_{1} t\right) \\
& \mp \sin (2 \beta)[Q \sin (2 \alpha)-U \cos (2 \alpha)] \sum_{m_{1}}^{\infty} \sum_{m_{2}}^{\infty} J_{m_{1}}\left(\delta_{10}\right) J_{m_{2}}\left(\delta_{20}\right) \cos \left[\left(m_{1} \Omega_{1} \pm m_{2} \Omega_{2}\right) t\right] \\
& -\cos (2 \alpha) \sin (2 \beta)[Q \sin (2 \alpha)-U \cos (2 \alpha)] \sum_{n_{1}}^{\infty} \sum_{n_{2}}^{\infty} J_{n_{1}}\left(\delta_{10}\right) J_{n_{2}}\left(\delta_{20}\right) \cos \left[\left(n_{1} \Omega_{1} \pm n_{2} \Omega_{2}\right) t\right] \\
& +V \sin (2 \beta) J_{0}\left(\delta_{10}\right) \sum_{m}^{\infty} J_{m}\left(\delta_{20}\right) \sin \left(m \Omega_{2} t\right) \\
& +V\left[\cos (2 \alpha) \sin (2 \beta) J_{0}\left(\delta_{20}\right)-\sin (2 \alpha) \cos (2 \beta)\right] \sum_{m}^{\infty} J_{m}\left(\delta_{10}\right) \sin \left(m \Omega_{1} t\right) \\
& +V \cos (2 \alpha) \sin (2 \beta) \sum_{m}^{\infty} \sum_{n}^{\infty} J_{m}\left(\delta_{10}\right) J_{n}\left(\delta_{20}\right) \sin \left[\left(m \Omega_{1} \pm n \Omega_{2}\right) t\right] \\
& \pm V \sin (2 \beta) \sum_{n}^{\infty} \sum_{m}^{\infty} J_{n}\left(\delta_{10}\right) J_{m}\left(\delta_{20}\right) \sin \left[\left(n \Omega_{1} \pm m \Omega_{2}\right) t\right],
\end{aligned}
$$


account of Eq. (1), we may, therefore, write the four required signals in the form:

$$
\begin{aligned}
I_{\mathrm{dc}} & =v_{1} I+v_{2} Q+v_{3} U, & I_{Q U 1} & =v_{4} Q+v_{5} U, \\
I_{Q U 2} & =v_{6} Q+v_{7} U, & I_{V} & =v_{8} V .
\end{aligned}
$$

In this equation, $v_{i}(i=1 \ldots 8)$ are constants that, in general, depend on the angular variables and parameters associated with the modulators $(\Omega$ and $\delta$ ).

However, these terms are not the actual signals recovered by the detector. This may be so for at least two reasons: (i) the quantum efficiency of the detector is not usually unity and (ii) the gain of the signal recovery electronics may be frequency dependent. Nevertheless, for a given frequency, and assuming that the detector is operating in a region of linear response, there is proportionality between the "theoretical" signals and the recorded signals. Thus, we may write

$$
\begin{aligned}
I_{\mathrm{dc}} & =c_{\mathrm{dc}} S_{\mathrm{dc}}, & I_{Q U 1} & =c_{Q U 1} S_{Q U 1}, \\
I_{Q U 2} & =c_{Q U 2} S_{Q U 2}, & I_{V} & =c_{V} S_{V},
\end{aligned}
$$

in which, for example, $I_{\mathrm{dc}}, S_{\mathrm{dc}}$, and $c_{\mathrm{dc}}$ represent the theoretical signal, the actual signal collected, and the corresponding proportionality constant, respectively. Equations (2) and (3) may be combined to give the following mätrix relation:

$$
\left(\begin{array}{c}
S_{\mathrm{dc}} \\
S_{Q U 1} \\
S_{Q U 2} \\
S_{V}
\end{array}\right)=\left(\begin{array}{cccc}
g_{1} & g_{2} & g_{3} & 0 \\
0 & g_{4} & g_{5} & 0 \\
0 & g_{6} & g_{7} & 0 \\
0 & 0 & 0 & g_{8}
\end{array}\right)\left(\begin{array}{c}
I \\
Q \\
U \\
V
\end{array}\right)=G\left(\begin{array}{c}
I \\
Q \\
U \\
V
\end{array}\right) .
$$

The matrix $G$ contains eight new constants, which are of the form $g=v / c$. The individual Stokes parameters can be found by a straightforward inversion of $G$, i.e.,

$$
\begin{aligned}
\left(\begin{array}{l}
I \\
Q \\
U \\
V
\end{array}\right) & =\left(\begin{array}{cccc}
k_{1} & k_{2} & k_{3} & 0 \\
0 & k_{4} & k_{5} & 0 \\
0 & k_{6} & k_{7} & 0 \\
0 & 0 & 0 & k_{8}
\end{array}\right)\left(\begin{array}{c}
S_{\mathrm{dc}} \\
S_{Q U 1} \\
S_{Q U 2} \\
S_{V}
\end{array}\right) \\
& =K\left(\begin{array}{c}
S_{\mathrm{dc}} \\
S_{Q U 1} \\
S_{Q U 2} \\
S_{V}
\end{array}\right),
\end{aligned}
$$

in which the elements $k_{i}(i=1,2, \ldots 8)$ in $K$ are the nonzero parameters. It is these elements that are determined experimentally through the calibration procedure. It should be noted that $k_{1}$ can only be found if the quantum efficiency of the photodetection system is known. However, for the majority of applications. this is not necessary and it is sufficient to determine the normalized calibration constants $k_{i} / k_{1}(i=2, \ldots 8)$ or effectively setting $k_{1}=1$.
A further relationship is admissible if the light is $100 \%$ polarized, namely,

$$
I^{2}=Q^{2}+U^{2}+V^{2} .
$$

Generally, the determination of $K$ will require a nonlinear regression to a set of experimental data.

\section{Experimental Considerations}

The experimental setup is shown in Fig. 1. The optical components comprise a diode laser source (wavelength $\lambda=670 \mathrm{~nm}$ ), Glan-Thompson polarizers (Karl Lambrecht) with an extinction coefficient of $10^{-6}$, two modulators (PEM-90, Hinds Instruments), and a silicon photodiode detector (Melles Griot). The modulators must operate at different frequencies $\left(\Omega_{1}=\right.$ $42 \mathrm{kHz}$ and $\Omega_{2}=50 \mathrm{kHz}$ in our experiment) so that fundamentals and harmonics may be distinguished. The modulation axis of PEM2 defines the $x$ axis of the laboratory coordinate system. The retarder used in the calibration is manufactured by Spectral Optics (8-400-IP Soleil-Babinet compensator). Finally, it should be noted that, because the laser emits highly linearly polarized light, there is a large variation in beam intensity as the polarizer is rotated. This is inconvenient in some instances and is rectified by placing the compensator (set for approximately quarter-wave retardation) between the laser and the polarizer. When elliptically polarized light is required, the polarizer and the compensator are interchanged: the fast axis of the latter is then oriented parallel to the laboratory $x$ axis and the polarizer rotated to $\pm 45^{\circ}$.

The ac components of the signal from the photodetector are measured with lock-in amplifiers (PerkinElmer 7265, Stanford Research Systems SR810DSP and EG\&G 5209) with either the PEM modulation frequencies of $\Omega_{1}$ and $\Omega_{2}$ or combinations thereof used as references. The dc component is measured with a high-precision electrometer (Keithley 6517). Although the use of three lock-in amplifiers is

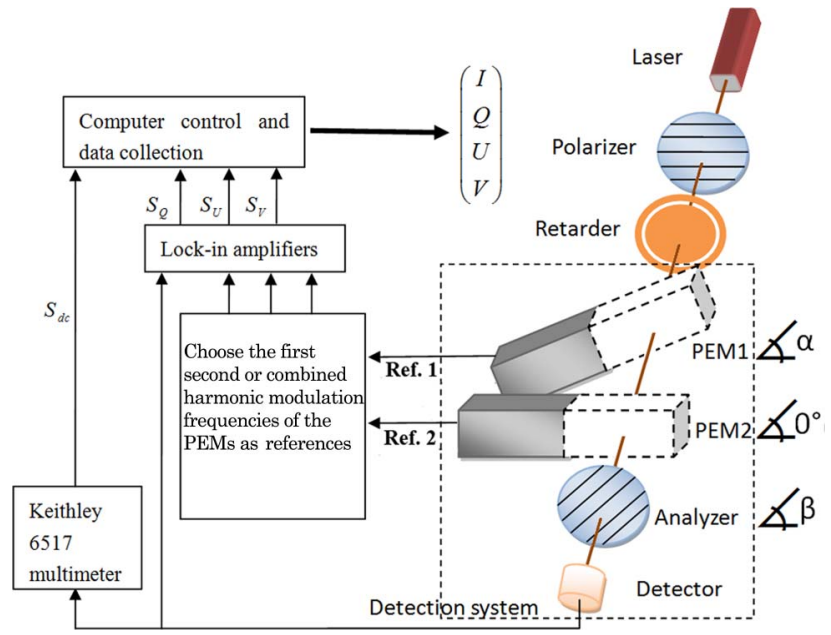

Fig. 1. (Color online) Schematic diagram of the dual-PEM-based Stokes polarimeter. 
preferable, a single amplifier may suffice to collect all three ac signals at each measurement point. It is straightforward to monitor fundamentals and harmonics, but combinatorial frequencies can also be measured either with custom-built electronics or by employing the "virtual reference" function of a lock-in amplifier with this facility (e.g., the PerkinElmer 7265). The virtual reference range of the lock-in is $0-120 \mathrm{kHz}$, which means that $\left(\Omega_{1}-\Omega_{2}\right)$ and $\left(2 \Omega_{1}-\Omega_{2}\right)$ are easily attainable, given that $\Omega_{1}=$ $42 \mathrm{kHz}$ and $\Omega_{2}=50 \mathrm{kHz}$. Owing to bandwidth limitations of the electronics, as well as the usually asymmetric terms associated with PEM1 and PEM2 in Eq. (1), the response of the measurement system to the $2 \Omega_{2}$ frequency may be less than that at the lower frequencies, which can lead to a somewhat attenuated $S_{Q U 2}$ signal. This has deleterious consequences because, in order to minimize errors [10], it is desirable that the eigenvalues of the product $\bar{K}^{T} K$ be of similar magnitude; $K^{T}$ is the transpose of $K$. The freedom to adjust the values of $\delta_{10}, \delta_{20}$, and $\beta$ enables $K$ to be tuned to possess such a property. A further advantage is the elimination of any setting errors associated with $\delta_{10}$ and $\delta_{20}$. In practice, the lack of constraint on $\delta_{10}$ and $\delta_{20}$ allows a PEM to be mounted with a slight tilt, thereby avoiding interference due to multiple reflections in the optical head.

\section{Calibration}

To find the elements of $K$, it is normal practice to impose additional experimental constraints. The most common procedure $[5,9]$ is to force the condition

$$
J_{0}\left(\delta_{10}\right)=J_{0}\left(\delta_{20}\right)=0
$$

by setting the retardation amplitude of both PEMs at $\delta_{10}=\delta_{10}=(2 \pi / \lambda)(0.383 \lambda)=2.4048 \mathrm{rad}$. Additionally, the number of constants in $K$ may be reduced by choosing particular values of $\alpha$ and $\beta$. Thus, for $\alpha=45^{\circ}$, we find from Eq. (1) that $\nu_{2}=\nu_{3}=\nu_{5}=\nu_{6}=$ 0 (and, similarly, for the constants $g_{i}$ and $k_{i}$ ). Effectively, the matrix $K$ is assumed diagonalized and the off-diagonal elements are assumed to be zero, and, hence, the calibration constants may be extracted by a standard linear regression method. (This is why we refer to the setup as the diagonalized case.) The only possible conditions permitting direct application of linear regression are $\alpha=45^{\circ}(\sin (2 \beta) \neq 0)$ and $\beta=45^{\circ}(\sin (2 \alpha) \neq 0)$ [23]. In the former case, there is a one-to-one correspondence between the signals received and the Stokes parameters; this is not true of the latter case. The calibration curves and corresponding linear relations for four different setups are shown in Figs. 2(a) and 2(b). At best, the error in any of the normalized constants does not exceed $2 \%$. Although this procedure is relatively straightforward, it does have disadvantages. The PEMs must be calibrated in situ and the values of $\delta_{10}$ and $\delta_{20}$ set accurately to ensure that $K$ is diagonalized. Also, we find that the measurements are affected by small variations in ambient temperature, which induce
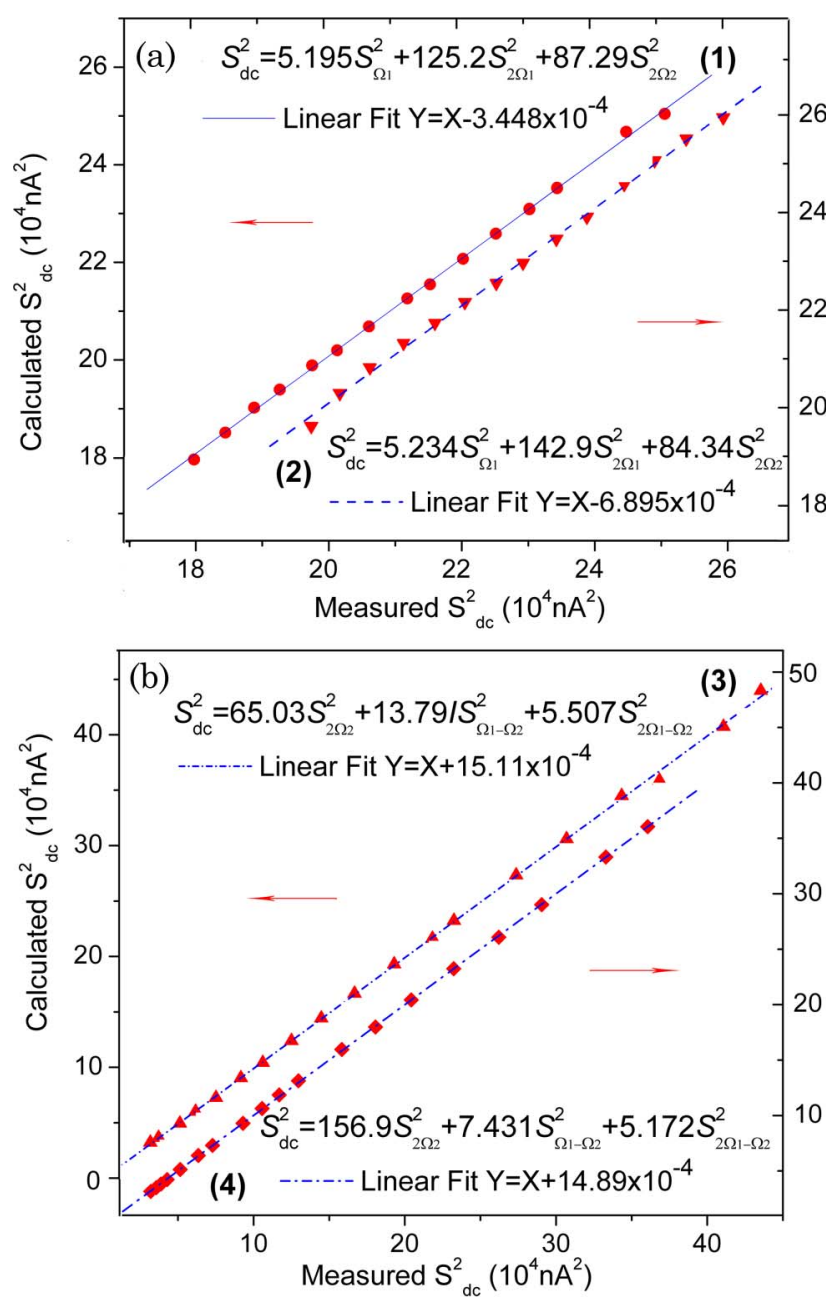

Fig. 2. (Color online) Calibration results for $J_{0}\left(\delta_{10}\right)=J_{0}\left(\delta_{20}\right)=0$. (a) Angle $\alpha$ is set at $45^{\circ}$ and $\beta$ is set at two arbitrary angles for curves (1) and (2). (b) Angle $\beta$ is set at $45^{\circ}$ and $\alpha$ is set at $30^{\circ}$ and $60^{\circ}$ for curves (3) and (4), respectively.

changes in the values of $\delta_{10}$ and $\delta_{20}$ (and, hence, the response of the PEMs).

As shown in Fig. 3, for a light beam of given polarization state, the signals calculated from Eq. (1) are strongly dependent on the settings of $\delta_{10}$ and $\bar{\delta}_{20}$. In the diagonalized case $\left(\Delta_{10}=\Delta_{20}=0.383 \lambda\right)$, the signals are sensitive to small fluctuations in retardation amplitude because of the large value of the first derivative of the Bessel functions with respect to the retardation. This situation may be ameliorated by relaxing the condition implied in Eq. (7). Thus, if we put $\Delta_{10}=\Delta_{20}=0.486 \lambda$, then $I_{\mathrm{dc}}, I_{Q U 1}$, and $I_{Q U 2}$ vary much less with retardation amplitude. By choosing this operating point, the signals become more resistive to ambient temperature fluctuation. However, $I_{V}$ is proportional to $J_{1}\left(\delta_{10}\right)$, which is not at its most stable when $\Delta_{10}=0.486 \lambda$. This makes it impossible to stabilize all signals simultaneously.

When Eq. (7) is not satisfied, the determination of $K$ becomes a nonlinear problem. We now outline a two-step method by which this may be done. Essentially, the calibration constants associated with the 


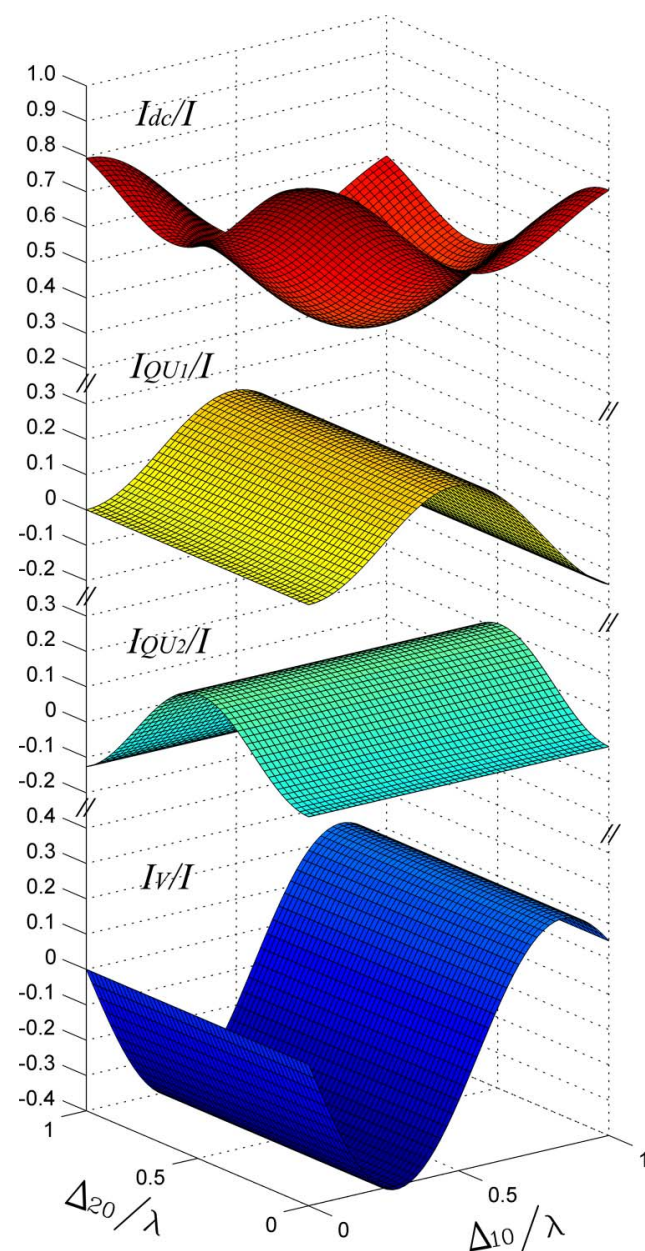

Fig. 3. (Color online) Normalized "theoretical" signals derived from Eq. (1) plotted as a function of $\Delta_{10}$ and $\Delta_{20}$ for an arbitrary polarization state.

linearly and circularly polarized components are found consecutively. For the first step, a quarterwave plate and polarizer are inserted between the source and the detection system: the light is now $100 \%$ linearly polarized and $V=0$. The analyzer may be placed at any angle subject to the condition that $\sin (2 \beta) \neq 0$, but PEM1 is set at an angle of $\alpha=45^{\circ}$. This means that $k_{5}=k_{6}=0$ and $K$ is effectively reduced to a $3 \times 3$ matrix with four nonzero normalized elements. Four special orientations are now chosen for the polarizer $\left(0^{\circ}, 45^{\circ}, 90^{\circ}\right.$, and $-45^{\circ}$ ), which allow simple relationships to be set up among the parameters $I, Q$, and $U$. These can

Table 1. Four Special Settings of the Polarizer Used to Determine the Relationships among $k_{1}, k_{2}, k_{3}, k_{4}$, and $k_{7}$

\begin{tabular}{cccc}
\hline & $\begin{array}{c}\text { Relationships among } \\
\theta\end{array}$ & $\begin{array}{c}\text { Derived } \\
\text { Expression }\end{array}$ & $\begin{array}{c}\text { Measured } \\
\text { Signals }\end{array}$ \\
\hline $0^{\circ}$ & $I=Q, U=0$ & $\frac{S_{d c}}{S_{Q U 1}}=\frac{k_{4}-k_{2}}{k_{1}}=a$ & $S_{\mathrm{dc}, 0}, S_{Q U 1,0}$ \\
$45^{\circ}$ & $I=U, Q=0$ & $\frac{S_{d c}}{S_{Q U 2}}=\frac{k_{7}-k_{3}}{k_{1}}=b$ & $S_{\mathrm{dc}, 45}, S_{Q U 2,45}$ \\
$90^{\circ}$ & $I=-Q, U=0$ & $\frac{S_{d c}}{S_{Q U 1}}=-\frac{k_{2}+k_{4}}{k_{1}}=c$ & $S_{\mathrm{dc}, 90}, S_{Q U 1,90}$ \\
$-45^{\circ}$ & $I=-U, Q=0$ & $\frac{S_{\mathrm{dc}}}{S_{Q U 2}}=-\frac{k_{7}+k_{3}}{k_{1}}=d$ & $S_{\mathrm{dc},-45}, S_{Q U 2,-45}$ \\
\hline
\end{tabular}

be used in conjunction with Eq. (5) to derive four equations connecting the calibration coefficients. These are written in the third column of Table 1.

The quantities $a, b, c$, and $d$ are ratios of two signals measured at each polarizer orientation. In turn, the equations in Table 1 can be used to obtain explicit expressions for the calibration constants normalized to $k_{1}$, namely,

$$
\begin{array}{ll}
\frac{k_{2}}{k_{1}}=-\frac{a+c}{2}, & \frac{k_{3}}{k_{1}}=-\frac{b+d}{2}, \\
\frac{k_{4}}{k_{1}}=\frac{a-c}{2}, & \frac{k_{7}}{k_{1}}=\frac{b-d}{2} .
\end{array}
$$

As already stated, the dc and $2 \Omega_{2}$ signals must be monitored in order to provide $S_{\mathrm{dc}}$ and $S_{Q U 2}$, respectively. According to Eq. (1), a choice of signal is available for $S_{Q U 1}$, but we have used $2 \Omega_{1}$, thus avoiding composite frequencies of the type $m_{1} \Omega_{1}+m_{2} \Omega_{2}$ (although these can be measured as mentioned in Section 3). As a demonstration of reproducibility, the first calibration step was repeated a number of times. The results are shown in Table 2. The accuracy with which the polarizer can be set to the same positions during each repetition of the procedure is determined by the precision of its rotary mount; 5 arc min in this case. However, we are aided by the fact that, at each setting, either $S_{Q U 1}$ or $S_{Q U 2}$ reduces to zero; the polarizer is adjusted to attain this condition.

To complete the calibration procedure, a SoleilBabinet compensator, set to produce an approximately quarter-wave retardation, is inserted between the polarizer and PEM1, as illustrated in Fig. 1. Varying degrees of circular polarization can now be generated by rotating the polarizer. The $\Omega_{2}$ frequency (recorded as $S_{V}$ ) is collected in addition to the dc, $2 \Omega_{1}$, and $2 \Omega_{2}$ signals. Assuming that the compensator does not introduce any significant depolarization, then the matrix $K$ in Eq. (5) effectively decouples the linear and circular polarized components. Using the calibration constants previously determined, the values of $I, Q$, and $U$ can be found from Eq. (5). The value of $V$ is now calculated from Eq. (6) and $k_{8} / k_{1}$ is obtained from the relation $V=\left(k_{8} / k_{1}\right) S_{V}$. Owing to the quadratic form of Eq. (6), the sign of the calibration constant $k_{8} / k_{1}$ remains unknown

Table 2. Normalized Calibration Constants Determined over Five Independent Sets of Measurements ${ }^{a}$

\begin{tabular}{ccccc}
\hline Calibration Set & $k_{2} / k_{1}$ & $k_{3} / k_{1}$ & $k_{4} / k_{1}$ & $k_{7} / k_{1}$ \\
\hline 1 & 0.47563 & 0.45475 & 2.09541 & 2.12227 \\
2 & 0.47468 & 0.45745 & 2.09398 & 2.12227 \\
3 & 0.47614 & 0.45733 & 2.09451 & 2.12249 \\
4 & 0.47454 & 0.45663 & 2.09425 & 2.12232 \\
5 & 0.47562 & 0.45689 & 2.09503 & 2.12294 \\
Mean & 0.47532 & 0.45661 & 2.09464 & 2.12246 \\
Standard deviation & 0.00069 & 0.00109 & 0.00058 & 0.00028 \\
Standard deviation $(\%)$ & 0.15 & 0.24 & 0.03 & 0.01 \\
\hline
\end{tabular}

${ }^{a}$ The PEM retardation amplitudes are set to $\Delta_{10}=\Delta_{20}=$ $0.486 \lambda$. 
(unimportant for many applications). The sign of $k_{8} / k_{1}$ is easily resolved, provided that (as is usually the case) the fast axis of the compensator is known.

In principle, $k_{8} / k_{1}$ can be determined from a single azimuthal orientation of the polarizer. However, the procedure was repeated for several settings, thus providing $k_{8} / k_{1}$ over a wide range of polarization states. Figure 4(a) plots the calculated values of $k_{8} / k_{1}$ against the ellipticity angle, $\chi$, defined as

$$
\chi=\frac{1}{2} \sin ^{-1}\left(\frac{V}{\sqrt{Q^{2}+U^{2}+V^{2}}}\right) .
$$

As $\chi$ decreases, $k_{8} / k_{1}$ becomes increasingly sensitive to small errors in $I, Q$, and $U$, resulting in a $7 \%$ deviation from the mean at $\chi=8^{\circ}$ (not shown on the graph). For this reason, only measurements made with an ellipticity angle greater than $15^{\circ}$ were used to determine the mean value of $k_{8} / k_{1}$.

To illustrate the general validity of the calibration procedure, the Stokes parameters were measured for a set of different polarization states of a fully polarized light beam (both linearly and elliptically polarized). The results are presented in Fig. 4(b) as $I^{2}$ versus $Q^{2}+U^{2}+V^{2}$. The gradient in the figure should remain constant (with a value of unity)
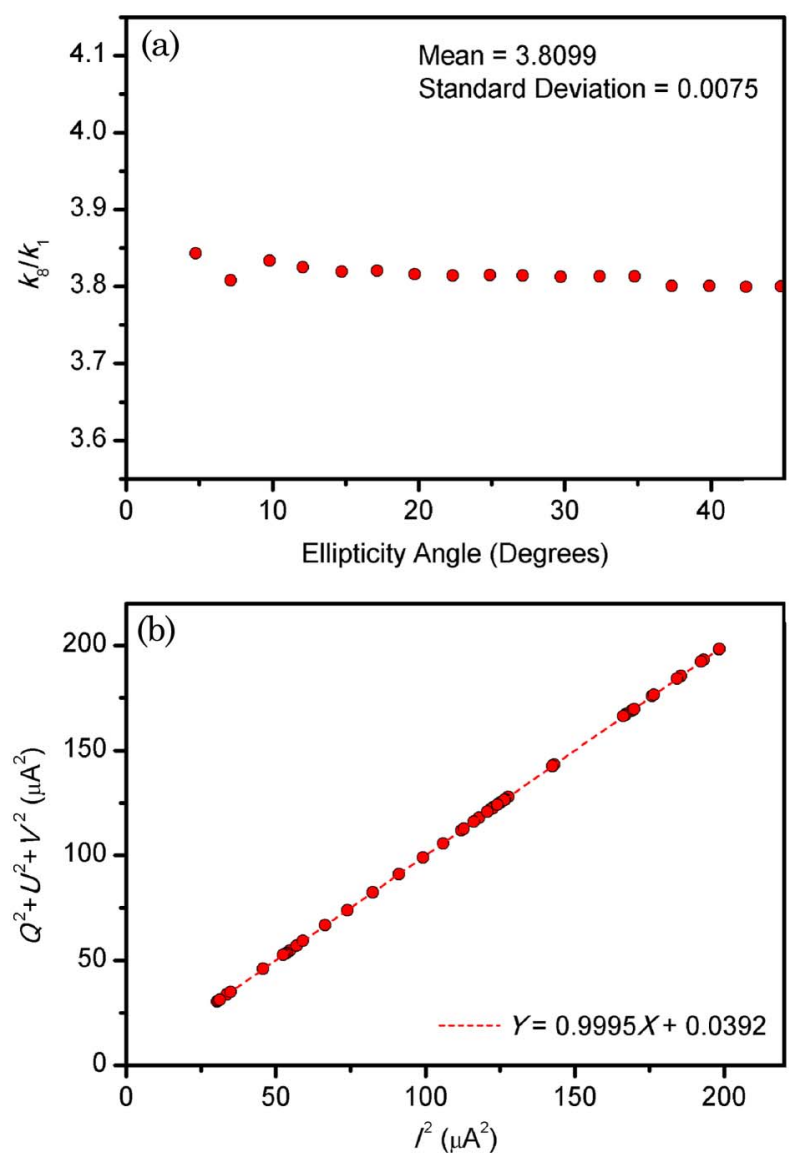

Fig. 4. (Color online) Tests for consistency for $\Delta_{10}=$ $\Delta_{20}=0.486 \lambda$. (a) Calculated value of $k_{8} / k_{1}$ as a function of ellipticity angle. (b) Plot showing the experimental validity of Eq. (6). regardless of the polarization state. The linear relationship demonstrates precisely this point, with the error in the gradient being no larger than $0.05 \%$. It should be noted that the construction of Fig. 4(b) involves use of all the calibration constants.

\section{Comparison of the Methods}

A brief critique of the relative merits of the diagonalized and nondiagonalized cases will now be given. The angle of polarization of plane polarized light was calculated for given orientations $\Psi$ of the polarizer. $\Psi$ is given by

$$
\Psi=\frac{1}{2} \tan ^{-1}(U / Q) .
$$

The degree of polarization was also determined. The mean error and maximum error in both these quantities $\left\langle\varepsilon_{P}\right\rangle,\left\langle\varepsilon_{\psi}\right\rangle, \varepsilon_{P \max }$, and $\varepsilon_{\psi \max }$ are presented in Table 3 for the three configurations under discussion, which were obtained from datasets of linearly polarized light of different polarization angles and elliptically polarized states with a varying degree of ellipticity. The columns headed $\Delta_{10}=\Delta_{20}=0.383 \lambda$ represent the situation where the retardations are taken as read from the PEMs. It is clear that the errors in the diagonalized case are significant and much larger than those incurred in the nondiagonalized case. This is because the readings of retardation indicated on the modulators do not represent their actual values. (In this specific example, in situ calibration showed that retardation amplitudes were out by $-6 \%$ and $-3 \%$ for the two PEMs used). In the columns headed $J_{0}\left(\delta_{10}\right)=J_{0}\left(\delta_{20}\right)=0$, the modulators were carefully calibrated in situ in order to ensure as far as possible the condition specified by Eq. (7). Now there is a dramatic improvement in the diagonalized case, but little difference between the diagonalized and nondiagonalized cases. Finally, results are shown for the setup where $\Delta_{10}=\Delta_{20}=0.486 \lambda$; there is little change compared with the procedure when $J_{0}\left(\delta_{10}\right)=J_{0}\left(\delta_{20}\right)=0$. The same behavior is also evident in Fig. 5 .

In view of the last observation, it might be argued that there is little advantage to be gained by moving away from a standard diagonalized procedure, especially so if the modulators are calibrated in situ. However, the nondiagonalized method offers a more robust approach. It has been found that the set point of $J_{0}\left(\delta_{10}\right)=J_{0}\left(\delta_{20}\right)=0$ is prone to drift as a result of changes in ambient temperature. In practice, the system would have to be recalibrated on a regular basis in an environment where the temperature changes by only a few degrees Celsius. Such instability can result in appreciable errors in the measured polarization state. For example, the ratio $U / Q$ used to calculate the azimuth angle, as given by Eq. (10), depends strongly on the ratio of the second-order Bessel functions $J_{2}\left(\delta_{20}\right) / J_{2}\left(\delta_{10}\right)$. By setting $\Delta_{10}=$ $\Delta_{20}=0.486 \lambda$, the first derivatives of $J_{2}\left(\delta_{10}\right)$ and $J_{2}\left(\delta_{20}\right)$ become zero and, consequently, the azimuth 
Table 3. Comparison of Mean and Maximum Errors in the Degree and Angle of Polarization

\begin{tabular}{|c|c|c|c|c|c|c|c|c|}
\hline & \multicolumn{4}{|c|}{ Diagonalized Method } & \multicolumn{4}{|c|}{ Nondiagonalized Method } \\
\hline & $\left\langle\varepsilon_{P}\right\rangle$ & $\varepsilon_{P \max }$ & $\langle\varepsilon \Psi\rangle$ & $\varepsilon_{\Psi \max }$ & $\left\langle\varepsilon_{P}\right\rangle$ & $\varepsilon_{P \max }$ & $\langle\varepsilon \Psi\rangle$ & $\varepsilon \Psi \max$ \\
\hline$\Delta_{10}=\Delta_{20}=0.383 \lambda$ & 0.0908 & 0.1823 & $0.1041^{\circ}$ & $0.1844^{\circ}$ & 0.0013 & 0.0038 & $0.0244^{\circ}$ & $0.0442^{\circ}$ \\
\hline$\Delta_{10}=\Delta_{20}=0.486 \lambda$ & - & - & - & - & 0.0011 & 0.0054 & $0.0242^{\circ}$ & $0.0511^{\circ}$ \\
\hline
\end{tabular}

measurement is less susceptible to small changes in PEM retardation. A factor of 5 improvement in the temperature stability of the azimuth measurement was achieved using this configuration.

Finally, we have investigated the possible effects of stray birefringence by introducing a constant retardation of $16 \mathrm{~nm}$ after the second PEM. All the calibration procedures coped well with a minimal increase in the measurement errors. Although, in principle, the precise impact of the stray birefringence can be analyzed by introducing an additional Mueller matrix to the formula [23], the resulting analytical expression is very complex. However, the residual retardation of the PEMs is claimed to be of the order of $0.1 \mathrm{~nm}$ and, therefore, we conclude that it has negligible impact on our results.
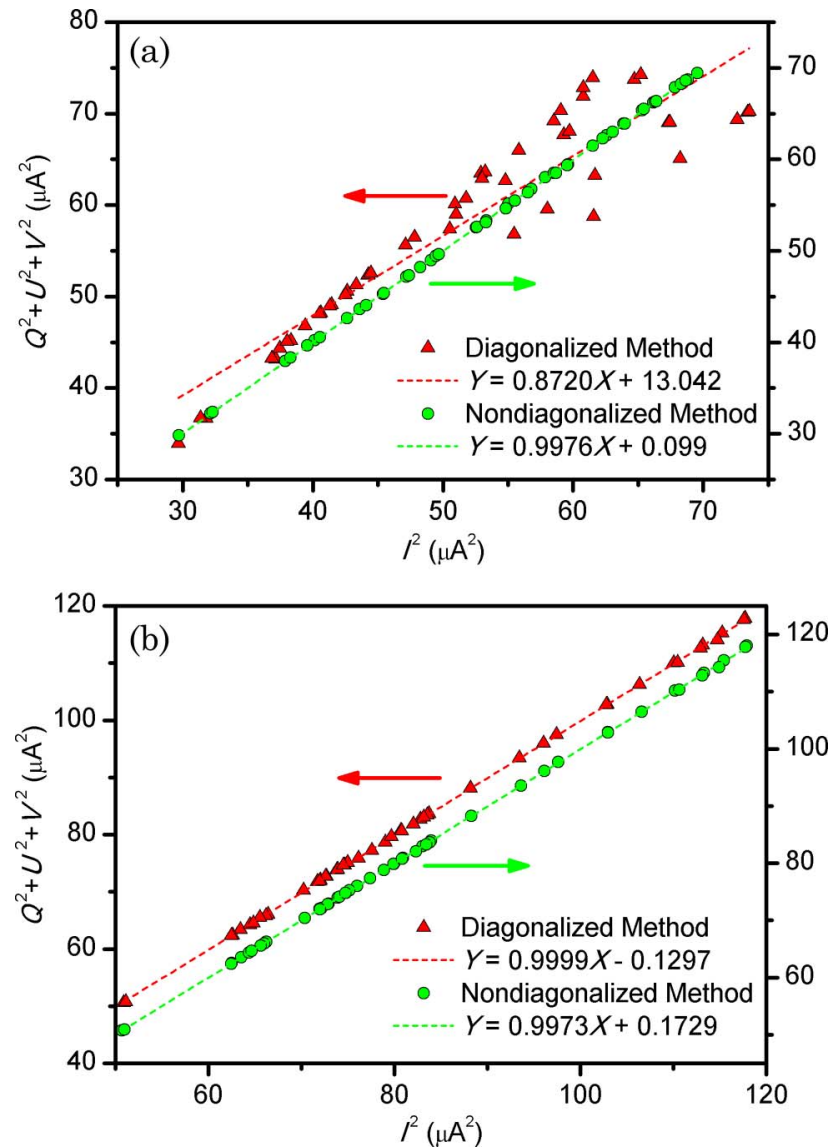

Fig. 5. (Color online) Comparison of experimental validity in terms of Eq. (6) for (a) setting $\Delta_{10}$ and $\Delta_{20}$ to the nominal value of $0.383 \lambda$ and $(\mathrm{b})$ ensuring the value of the zero-order Bessel function to be zero.

\section{Measurement of Magneto-Optical Properties}

To illustrate the sensitivity of the system, we have investigated the magneto-optical Faraday effect of a CoPt multilayer film with a strong magnetic easy axis perpendicular to the film plane. The sample comprises a $10 \mathrm{~nm} \mathrm{Pt}$ seed layer followed by 15 bilayers of $\mathrm{Co}(0.4 \mathrm{~nm})$ and $\mathrm{Pt}(1 \mathrm{~nm})$, giving a total film
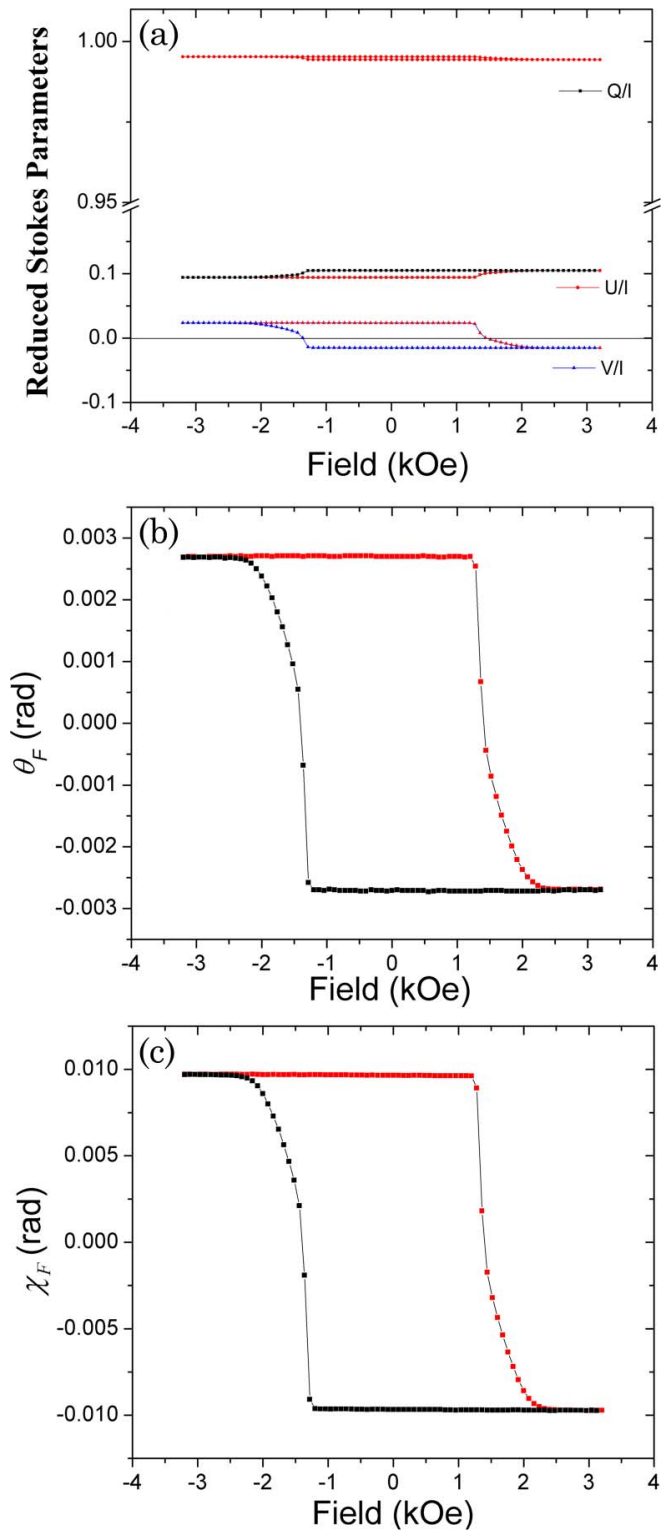

Fig. 6. (Color online) (a) Reduced Stokes parameters, (b) Faraday rotation angle, and (c) ellipticity angle of a CoPt multilayer measured with the calibrated dual-PEM system. 
thickness of $31 \mathrm{~nm}$ (including the seed layer) deposited on a glass substrate. The experimental setup is identical to that of Fig. 1, except that the sample and electromagnet are substituted for the compensator. The reduced Stokes parameters, such as $Q / I$, are plotted in Fig. 6(a), which shows that the fieldinduced changes to the polarization state are relatively small.

The Faraday rotation angle, $\theta_{F}$, is calculated using Eq. (10) and the ellipticity angle, $\chi_{F}$, is found from the Eq. (9). These quantities are shown in Figs. 6(b) and 6(c). They show that, whereas the accuracy in the determination of the absolute angles of polarization from $Q$ and $U$ is usually of the order of $0.05^{\circ}$, the sensitivity to changes in polarization state is several orders of magnitude superior. The typical noise level in the polarization angle, $\theta_{F}$, is about 0.06 arc sec. This is obtained without any optimization of the detector-signal recovery electronics, implying a strong potential for further improvement.

\section{Conclusion}

The retardation amplitude of a PEM is typically factory calibrated to a few percent, and it is often operated at a slightly tilted angle with respect to the laser beam to avoid the interference of multiple reflections. However, both are likely to introduce errors in the precise setting of the retardation amplitude, such that $J_{0}\left(\delta_{10}\right)=J_{0}\left(\delta_{20}\right)=0$, and may cause significant error in the Stokes parameter measurements when the diagonalized method is employed. A more general methodology for a dual-PEM system that permits the relaxation of the fixed retardation amplitudes of the PEMs has been developed. This offers more flexibility and robustness in the detection of polarization states. Although it is not possible to optimize all measured signals simultaneously, the theory can indicate the ideal choice of experimental setup for any given application, for instance, to maximize or improve temperature stability of the desired signals by avoiding $J_{0}\left(\delta_{10}\right)=J_{0}\left(\delta_{20}\right)=0$.

In conjunction with the theory, a two-step procedure that gives the calibration constants to an accuracy of better than $1 \%$ and also preserves their sign has been devised. This means that the signs of the Stokes parameters are known unambiguously. The only preset or known value required is $\alpha$, the angle between the two PEMs. The calibration procedure may be verified with simple tests for self-consistency by the application of Eq. (6) using a coherent light source with a range of different polarization states. Indeed, if necessary, a refinement of the calibration constants can be sought by use of a multiparameter nonlinear optimization procedure, where the constraint on $\alpha$ can also be relaxed. While the latter details will be published elsewhere, our conclusion is that the two-step procedure will suffice for the majority of applications.

We thank E. Hill of Manchester University for providing the CoPt sample. T. Shen acknowledges helpful discussions with H. L. Le, University of Not- tingham. W. Guan thanks the Overseas Research Students Awards Scheme (ORSAS) and the University of Salford for a research studentship. P. J. Cook acknowledges the Engineering and Physical Sciences Research Council (EPSRC) for a research studentship. We thank P. J. Grundy for his interest and encouragement and M. Clegg for technical support.

\section{References}

1. M. Billardon and J. Badoz, "Modulateur de birefringence," C. R. Acad. Sci. Ser. B 262, 1672-1675 (1966).

2. M. Born and E. Wolf, Principles of Optics, 7 th ed. (Cambridge U. Press, 1999).

3. D. H. Goldstein and E. Collett, Polarized Light, 2nd ed. (Marcel Dekker, 2003).

4. D. J. Diner, A. Davis, B. Hancock, G. Gutt, R. A. Chipman, and B. Cairns, "Dual-photoelastic-modulator-based polarimetric imaging concept for aerosol remote sensing," Appl. Opt. 46, 8428-8445 (2007).

5. B. L. Wang, J. List, and R. Rockwell, "A Stokes polarimeter using two photoelastic modulators," Proc. SPIE 4819, 1-8 (2002).

6. G. E. Jellison and F. A. Modine, "Two-modulator generalized ellipsometry: theory," Appl. Opt. 36, 8190-8198 (1997).

7. B. L. Wang, "Linear birefringence measurement instrument using two photoelastic modulators," Opt. Eng. 41, 981-987 (2002).

8. M. Kuldkepp, N. C. Hawkes, E. Rachlew, and B. Schunke, "Accurate polarization measurements with a dual photoelastic modulator," Appl. Opt. 44, 5899-5904 (2005).

9. G. R. Boyer, B. F. Lamouroux, and B. S. Prade, "Automaticmeasurement of the Stokes vector of light," Appl. Opt. 18, 1217-1219 (1979).

10. E. Compain and B. Drevillon, "High-frequency modulation of the four states of polarization of light with a single phase modulator," Rev. Sci. Instrum. 69, 1574-1580 (1998).

11. Y. W. Liu, G. A. Jones, Y. Peng, and T. H. Shen, "Generalized theory and application of Stokes parameter measurements made with a single photoelastic modulator," J. Appl. Phys. 100063537 (2006).

12. M. Mujat and A. Dogariu, "Real-time measurement of the polarization transfer function," Appl. Opt. 40, 34-44 (2001).

13. B. L. Wang, "Measurement of circular and linear birefringence in chiral media and optical materials using the photoelastic modulator," Proc. SPIE 3535, 294-302 (1999).

14. S. M. Jordan and J. S. S. Whiting, "Detecting two components of magnetization in magnetic layer structures by use of a photoelastic modulator," Rev. Sci. Instrum. 67, 4286-4289 (1996).

15. Y. Shindo, "Application of polarized modulation technique in polymer science," Opt. Eng. 34, 3369-3384 (1995).

16. J. C. Canit and J. Badoz, "Photoelastic modulator for polarimetry and ellipsometry,” Appl. Opt. 23, 2861-2862 (1984).

17. K. W. Hipps and G. A. Crosby, "Applications of the photoelastic modulator to polarization spectroscopy," J. Phys. Chem. 83, 555-562 (1979).

18. C. F. Wong, "Birefringence measurement using a photo-elastic modulator," Appl. Opt. 18, 3996-3999 (1979).

19. F. A. Modine, G. E. Jellison, and G. R. Gruzalski, "Errors in ellipsometry measurements made with a photo-elastic modulator," J. Opt. Soc. Am. 73, 892-900 (1983).

20. J. Badoz, M. P. Silverman, and J. C. Canit, "Wave-propagation through a medium with static and dynamic birefringencetheory of the photoelastic modulator," J. Opt. Soc. Am. A 7, 672-682 (1990) 
21. J. C. Kemp, G. D. Henson, C. T. Steiner, and E. R. Powell, "The optical polarization of the sun measured at a sensitivity of parts in 10-million," Nature 326, 270-273 (1987).

22. S. N. Jasperson and S. E. Schnatterly, "An improved method for high reflectivity ellipsometry based on a new polarization modulation technique," Rev. Sci. Instrum. 40, 761-767 (1969).
23. W. Guan, G. A. Jones, Y. W. Liu, and T. H. Shen, "The measurement of the Stokes parameters: a generalized methodology using a dual photoelastic modulator system," J. Appl. Phys. 103, 043104 (2008).

24. J. C. Kemp, "Piezo-optical birefringence modulators-new use for a long-known effect," J. Opt. Soc. Am. 59, 950-954 (1969). 